\title{
Securing the Operation of Socially Critical Systems from an Engineering Perspective: New Challenges, Enhanced Tools and Novel Concepts
}

\author{
Wolfgang Kröger ${ }^{1}$
}

Received: 4 January 2017/Accepted: 23 January 2017/Published online: 10 February 2017

(C) Springer International Publishing Switzerland 2017

\begin{abstract}
Large-scale infrastructure systems, providing essential goods and services to our societies and economies, are witnessing tighter integration and coupling, notably by the sheer ubiquitous use of cyber-physical systems for integration, communication, and control. Putting the electric power system in focus, the pertinent supervisory and data acquisition systems are not only running through major changes allowing for increased levels of communications but also exposing them to new (cyber) threats. Furthermore, humans are an essential part of these systems interacting with them and developing them into a "system of socio-technical systems". In theory, and evidenced by past blackouts, power grids show complex behaviors and a strong influence of contextual factors. While the deterministic "N-1 security principle" has been successful in ensuring high performance of the European grid, it has been deemed insufficient to cope with multiple failures, cascading grid tripping scenarios, and non-technical factors and to capture a widened spectrum of threats. Thus, as traditional models often reach their limits, advanced modeling and simulation techniques are necessary, which are partly available and applied. Moreover, past disasters prove the need of focusing on "after shock behavior" and precaution against unanticipated events by amplifying absorptive, adaptive, and recovery capabilities. This shift towards increased resilience requires substantial conceptual work and the further development of analytical tools. Here the latest achievements and future challenges in this field of active research, aiming at securing the operation of those systems, are highlighted.
\end{abstract}

Keywords Critical infrastructure · Complexity · Power blackouts · Modeling power grids $\cdot$ Resilience

Wolfgang Kröger

kroeger@ethz.ch

1 ETH Zürich, SEC E6, Scheuchzerstrasse 7, 8092 Zürich, Switzerland 


\section{Introduction}

Large-scale, human-made systems, called infrastructures, are composed of many components, interacting in multiple ways in a networked structure. They are often structurally and technologically evolving, which all leads to structural and dynamic complexity. The components function synergistically to produce and provide goods and services. The infrastructure systems have undergone tighter integration and growing interdependencies and will (probably to an increasing extent) continue to do so. Furthermore, humans are an essential part of these systems as they interact with them both individually, e.g., as operators and managers, and collectively, e.g., as users, which turns them into socio-technical (engineered) infrastructure systems (Nan and Sansavini 2017). As commonly agreed, an infrastructure is termed "critical" if its incapacity or destruction has a significant impact on health, safety, security, economics, and social well-being (Council of the European Union 2008).

Most recent and foreseeable developments are driven by integration and control via digital information and communication technology (ICT)—with both sophisticated hardware and software. Keeping this in mind we are urged to comprehend critical infrastructures as a system of coupled systems that modern societies increasingly depend on. Besides indisputable benefits, this pervasive use of ICT, including the Internet, as "host technology" induces a higher risk of potential common cause failures and malicious attacks while the closer gearing of systems may trigger cascades across boundaries in case of failures in single systems.

As many significant publications are available dealing with critical infrastructures and related characteristics in general, e.g., (Zio 2016), we focus on the electrical grid as one of the most essential critical infrastructures, while being a crucial part of a broader "system-of-systems".

Section 2 will address monitoring and control issues while in Sect. 3-after a short outline of the European Transmission system-major changes and challenges are sketched. The following sections allude to best practice and some deficits and provide information about major blackouts and point to the importance of contextual factors. Section 6 presents challenges to modeling of such systems including human actions and Sect. 7 offers some advanced methods and conceptual frameworks to capture their complex behaviors before addressing issues of malicious attacks in Sect. 8. Finally, the evolving concept of resilience and some early guiding principles are introduced (Sect. 9), followed by concluding remarks.

\section{Control of the Electrical Power Grid}

Besides more obvious elements such as lines, poles, and substations, Industrial Control Systems (ICS) are provided to monitor and support reliable electricity transport and distribution; the largest subgroup of ICS are Supervisory and Data Acquisition (SCADA) systems. While SCADA systems have a long tradition as dedicated, singular systems they are nowadays standard technologies, ubiquitously using off-theshelf hardware and software. Moreover, they are highly interconnected with other 
corporate networks, including those for business (i.e., energy trading) operation management and the Internet (see Fig. 1 for SCADA ISA95 standard architecture).

These present-and potentially further developed-systems allow for increased levels of communications (e.g., remote access, increased automation, improved (nearreal-time) monitoring, and supervision) but they are also exposed to new and traditional threats (European Network and Information Security Agency (ENISA) 2016). As they are related to an essential critical infrastructure, they have been attacked and are likely to be increasingly attacked in the future (SANS Institute 2016).

\section{Challenges to the European Transmission System}

The European Transmission System (ETS) is highly meshed and evolving. It entails five synchronous large area networks with about $314,333 \mathrm{~km}$ of high-voltage lines, is operated by 41 Transmission System Operators (TSOs) from 34 countries, and serves 534 million citizens with an annual electricity consumption of about 3278 TWh (all numbers as of 2015, http://www.entso-e.eu). To ensure security of supply and reliable operation, the ETS needs to be protected against cascade tripping, voltage or frequency collapse, and loss of synchronism.

The ETS has been subject of major changes and associated challenges:

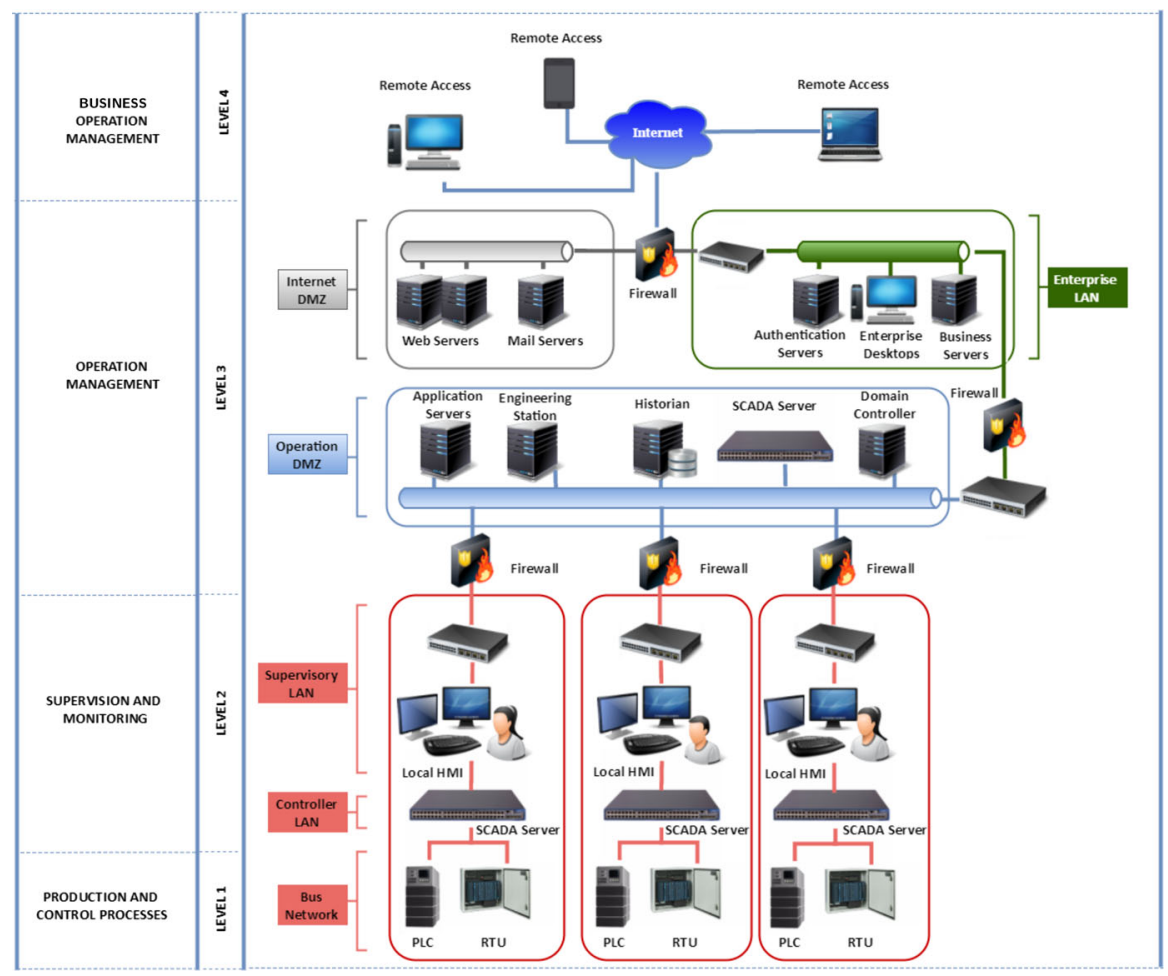

Fig. 1 ISA95 levels applied to a SCADA architecture (http://www.isa-95.com) 
- Replacement of "monopolies" to intricate (unbundled) market structure with a shift of responsibility for reliable power supply from single industries to national authorities.

- Push of operation modes closer to limits, i.e., economically motivated operations beyond initial design parameters.

- Integration of a rapidly increasing share of intermittent asynchronous renewable energy sources - notably wind and solar with a share of $17 \%$ of annual power production that are highly dispersed, usually abundant in scarcely populated areas, and often available during low demand periods thus requiring both massive power transfers over long distances as well as peak smoothing strategies.

- Increasing volumes of cross-border power exchange, nowadays slightly above $15 \%$, as well as of short-term trading.

- Increasing "smartness" and user involvement due to pervasive use of ICT, selfsustaining areas, and transfer of control functions from central units to decentralized private users while at the same time a coexistence of "old" and "new" technologies and structures must be managed.

- Dual role of end users as electricity consumers and generators trend to partial local autonomy.

- Widening of the spectrum of threats and hazards to malicious cyber and physical attacks and extreme weather conditions, both with increasing frequency and severity; moreover, many systems and components suffer from aging, lack of adequate investment, and decreasing redundancy/reserves.

Furthermore, due to excellent performance of the whole supply chain, electricity is widely regarded as steadfast common good; potential major blackouts lack public awareness and are met with complacency.

\section{Best Operational Practice}

The European Transmission System (ETS) is managed according to the instructions in the UCTE $^{1}$ (now ENTSO-E ${ }^{2}$ ) Operational Handbook (European Network of Transmission System Operators for Electricity (ENTSOE) 2004). This is a comprehensive collection of operational principles, technical standards, and recommendations for Transmission System Operators (TSOs) in continental Europe being responsible for their own network. To ensure security against sudden disturbances in operational planning and real-time operation, the $\mathrm{N}-1$ principle is of major importance (see Box 1).

\footnotetext{
${ }^{1}$ Union for the Co-ordination of Transmission of Electricity. It coordinated the operation and development of the electricity transmission grid for the Continental European synchronously operated transmission grid, providing a platform to participants of the Internal Electricity Market. See https://www. entsoe.eu/news-events/former-associations/ucte/Pages/default.aspx.

2 European Network of Transmission System Operators. It consists of 42 TSOs from 35 countries across Europe and was established and given legal mandates by the EU's Third Legislative Package for the Internal Energy Market in 2009.
} 
Box 1 Best practice tools to ensure security of the European Transmission System: N-1 principle and associated measures

In Europe, the UCTE (now ENTSO-E) Operation Handbook (European Network of Transmission System Operators for Electricity (ENTSOE) 2004) provides binding principles, standards and recommendations to help Transmission System Operators (TSOs) manage their own network and ensure interoperability among them

The N-1 principle requires that after a failure of a single element the remaining must be capable to accommodate the change of flows and avoid cascading effects

Each TSO has to develop a list of contingencies: (a) normal (a line break), (b) exceptional (two electricity lines on same tower) and (c) out of range (tower with more than two lines)

Internal and external events need to be included in security calculations, mandatory for type (a) and (b) contingencies, while (c) compromises losses of elements with very low probability, for which security calculations are not mandatory

The N-1 framework has been successful in ensuring high performance of transmission systems, if properly implemented (!), but deems insufficient to cope with multiple failures and tripping scenarios

This mainly deterministic framework has been successful in ensuring high performance of the ETS, proper implementation provided. However, it is insufficient to cope with multiple failures, tripping scenarios, and strong influence of operational/contextual factors. These effects go beyond $\mathrm{N}-1$ security requirements. but play a significant role in major blackouts, as we experienced in the past.

\section{Learning from Major Blackouts}

Table 1 comprises a list of worldwide major blackouts for the time period from mid1999 to the end of 2015. They are termed "major" as the outages were (a) not planned (at the date of occurrence) by the service provider, (b) affected at least 1000 people, and (c) lasted at least $1 \mathrm{~h}$. In total, roughly 100 events happened, conforming to the above criteria (visit Wikipedia 2003) out of which 25 events have been selected for further evaluation and characterization, of the main causes in particular. This list emphasizes not only the (unsurprisingly) dominating role of natural hazards as triggers (at least 7 out of 25 events) but also the (astonishingly) the important role of organizational/contextual factors (roughly 6 out of 25), since only one outage was triggered by purely technical failure and additional two outages by fire. It is important to note that we experienced-besides one potential military attack-two cyber attacks on power grids, the worst just recently on three distribution grids in the Ukraine.

Regarding the latter, on December 24, 2015, coordinated cyber attacks were directed at the regional distribution level $(110 \mathrm{kV}$ and below) against three regional electricity distributors ("oblenergos") in the Ukraine. The attacks were executed within $30 \mathrm{~min}$ of each other and impacted 225,000 customers. The distributors were able to restore service quickly by moving to manual operations after an outage window lasting several hours. The motive and sophistication of this power grid attack is consistent with a highly structured and resourced actor who demonstrated a 


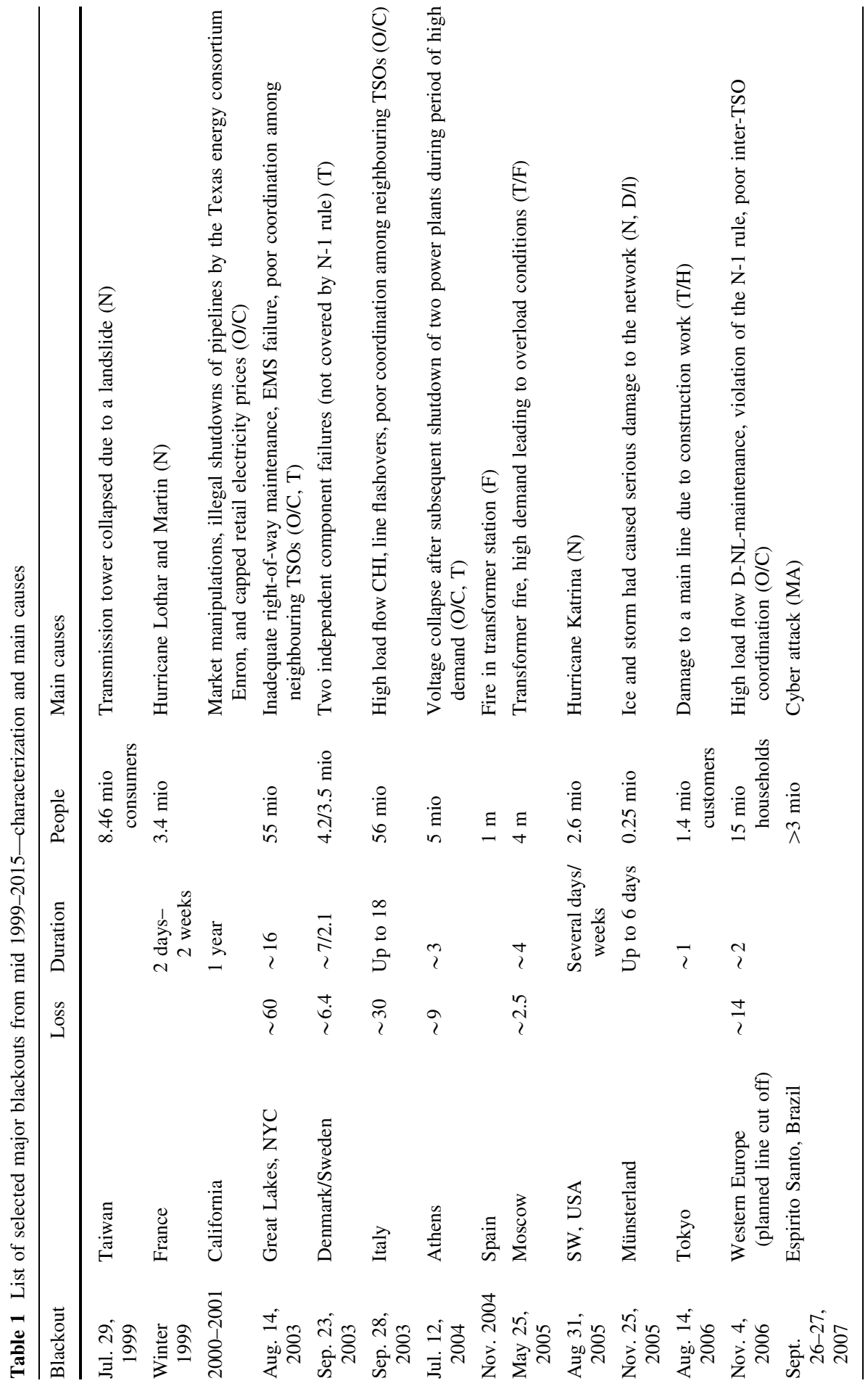




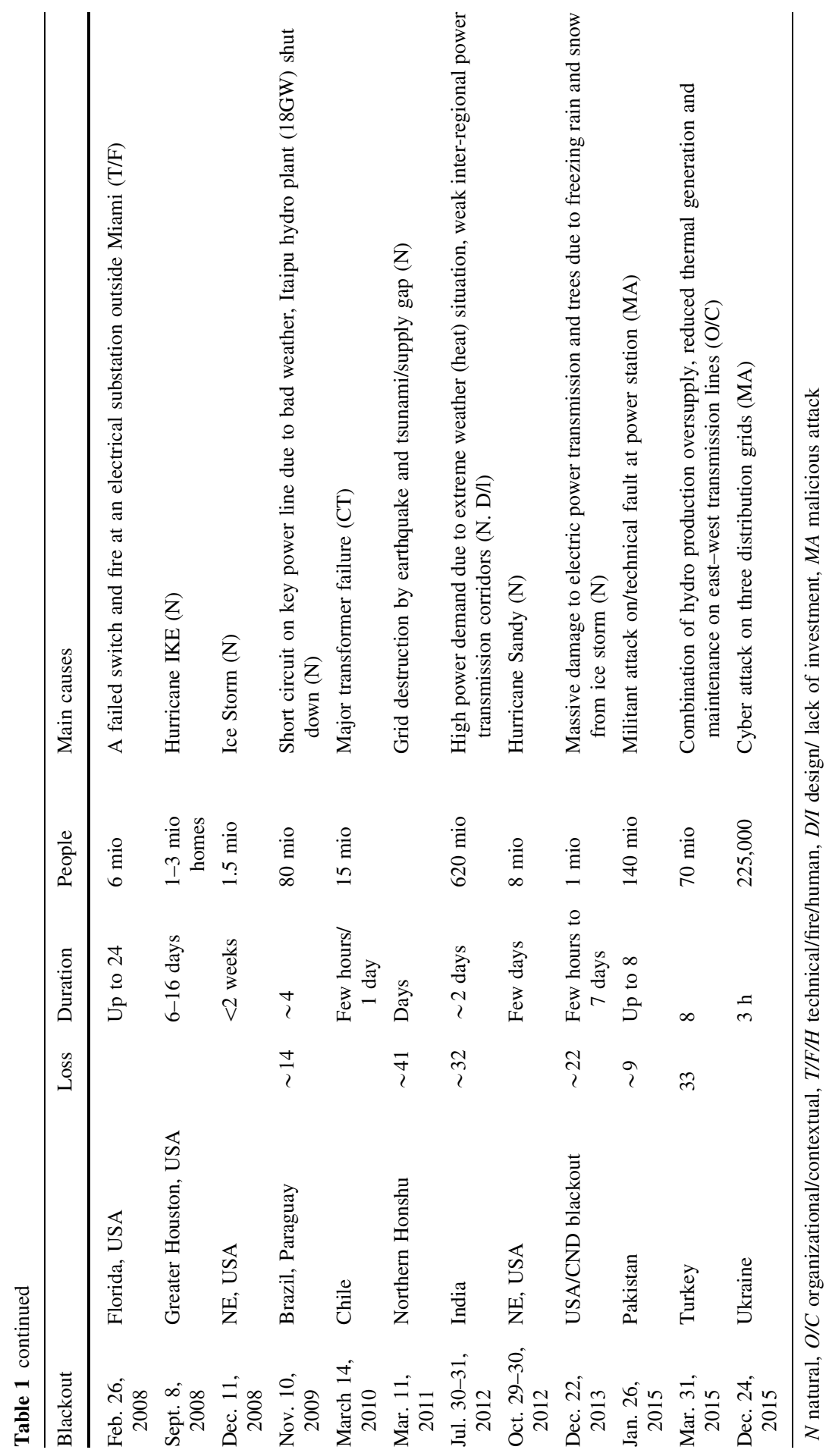




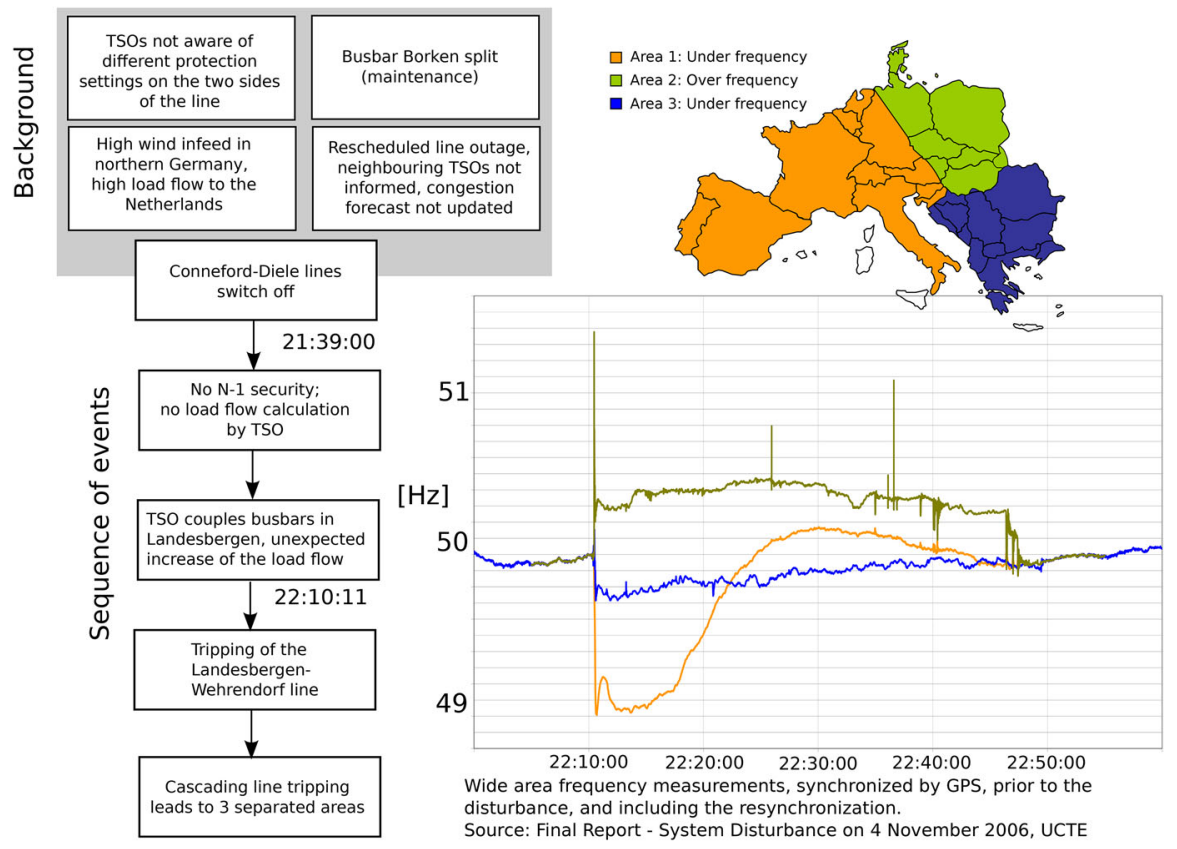

Fig. 2 Disturbance of the European Transmission System on 4 November, 2004-background, sequence of events and wide area frequency measurement versus time

variety of capabilities including not only the choice of tools but also their ability to perform long-term reconnaissance operations to learn the environment and execute a highly synchronized, multistage, multi-site attack (SANS Industrial Control Systems (SANS ICS), Electricity Information Sharing and Analysis Center (EISAC) 2016).

To further improve our understanding and derive challenges to enhanced modeling of power transmission systems we look closer into another example illustrating organizational/contextual factors interplaying with human and technical factors as types of outage triggering causes, i.e., the Western Europe transmission system split on November 4, 2006. Triggered by a planned but re-scheduled switching-off of the 380-kV Diele-Conneford line over the River Ems in northern Germany (to let an inland built cruise liner pass), tripping of many high voltage lines followed, finally dividing the continental European grid into three islands with significant power imbalances in each island (see Fig. 2). The induced frequency drop in the Western area caused an interruption of supply for 15 million households; the full resynchronization was completed within $38 \mathrm{~min}$, and the normal situation in all European countries was re-established in less than $2 \mathrm{~h}$. The investigations identified two root causes (ENTSOE 2006): first, the actual evaluation of N-1 secure conditions was not based on results of a numerical analysis; only an empirical evaluation of the situation was performed so that the N-1 criterion was not fulfilled. Second, the regional inter-TSO co-ordination was inappropriate and no specific attention was given to the fact that the protection devices have different settings on 
both sides of one line, the Landesbergen-Wehrendorf line. Furthermore, five critical factors were identified, all of them organizational/contextual by type. Moreover, nature played its part: when the lines were switched off at 21:39 there were strong winds in northern Germany, causing high load in-feed, notably to The Netherlands. This event constitutes the most severe disturbance in the history of UCTE.

These relatively few events put purely technical failures in the back seat but demonstrate the crucial role of common cause aerial events, like extreme weather situations and earth/seaquakes with induced tsunamis, and the importance of "soft factors", notably organizational/contextual. Meanwhile, extreme weather situations are expected to increase in both frequency and severity due to climate changes, since the spectrum of threats has extended to cyber attacks. This needs to be kept in mind when trying to understand and characterize the behavior of electric power grids (and most socially critical infrastructures) and to better secure their operation.

\section{Challenges to Modeling of Socio-Technical Critical Systems}

As demonstrated by many major disturbances (the system split of 2006 in particular), large-scale, multi-layered electric power grids show the emergence of unprecedented complex behaviors as a hallmark, that is, with the potential of nonlinearities, dynamics, feedback loops, and even regime shifts; small changes of initial conditions can trigger cascades and accelerate to big global effects (system breakdown). Power grids weakly depend on other critical infrastructures besides ICT, notably for black starts (Kröger and Sansavini 2016). Realistic efforts and research, respectively, to better understand the behavior of those systems in response to disruptive events are challenged by their key characteristics and need to take the operational and organizational context into account as stated before.

Unfortunately, it is deemed infeasible to build lab-scale experimental setups that expose the entire system to failures and attacks. To restrict studies to empirical investigations of failures and outages is also unsatisfactory as the events are too rare because of high design and operational standards. Therefore, we have to resort to models and simulations - that can adequately capture the system aspects, the system behavior in response to a wide set of hazards/threats/failures, and underlying physics-taking advantage of quite limited empirical observations, like those addressed before. To further elaborate the set of challenges, the models have to follow a systems approach as the collective behavior is more than (different from) the sum of individual behaviors and copes with dynamics. Here the use of classical methods based on decomposition, like fault trees, and quasi-static causal chains, like forward binary event trees, is limited.

\section{What Science Can Offer}

Advanced modeling and simulation approaches are available and have been applied to complex networks including the electric power grids and, more recently, also to "smart grids". Based on surveys (Pagani and Aiello 2013) and own research, it 
became obvious that no single modeling approach "that captures it all" is available or even feasible; a conceptual framework was proposed (Eusgeld and Kröger 2008) to bring all system aspects together and to take advantage of the diverse capabilities of the modeling and simulation approaches currently available or under development.

Complex network theory (CNT) has become pervasive, aiming to understand the structure of interactions of a plethora of components and to check vulnerability by removal of components (nodes). Fundamentally, it transforms a real system (e.g., a power grid) into a graph with nodes (stations, substations) and links (lines), builds the adjacency matrix and measures drop in performance by a group of structural or centrality features such as path length, node degree distribution, betweenness, clustering coefficient, and size of largest component (Zio and Sasavini 2008). These descriptors (metrics) are used to infer the reliability and robustness of the power system and to offer interesting insights, e.g., that most power grids have a highly homogenous structure with hubs making them robust against random failures but, in return, vulnerable against targeted attacks (see Fig. 3 for illustration).

Beyond useful information about structural properties and topological vulnerabilities, it provides information at the level of system functionality when assuming that it can be approximated and captured by the connectivity pattern of its components. For example, the size of the largest connected component of a graph is used as a proxy for the level of the system performance, and it is hypothesized that

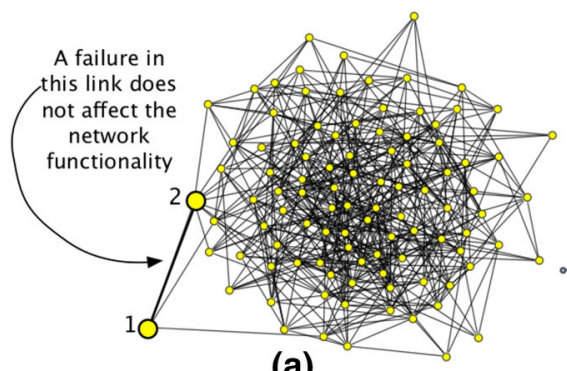

(a)

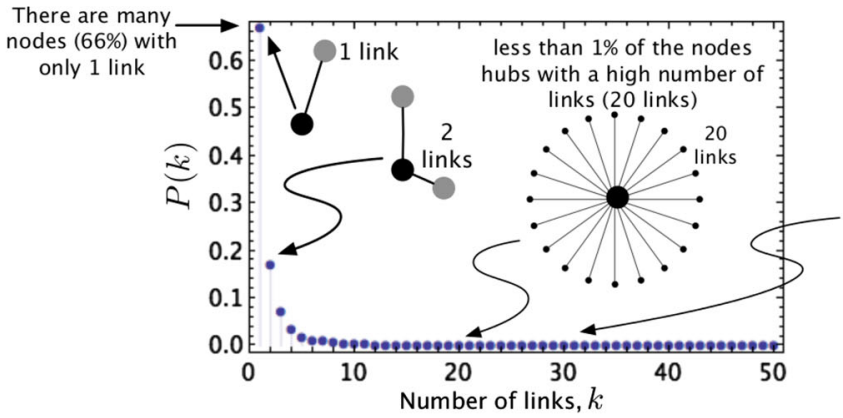

(c)

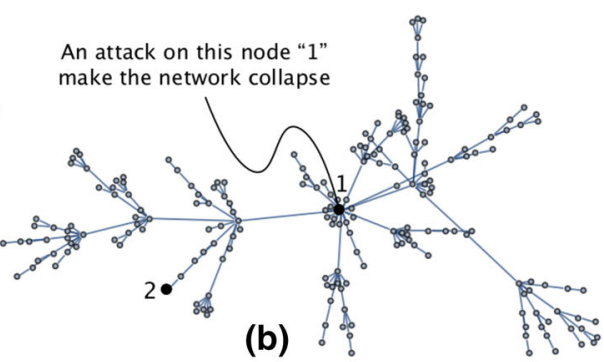

30 links

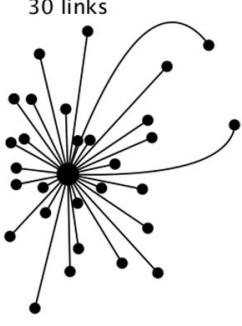

Hetereogeneity is the key property!

Fig. 3 Illustration of complex network theory: a example of robust network; $\mathbf{b}$ example of a scale-free network, vulnerable to attacks on nodes with many links; $\mathbf{c}$ node degree probability distribution function of a network similar to that in b (Cuadra et al. 2015) 
catastrophic transitions in system operations are connected to the sudden disappearance of that "giant component" when a fraction of its links is removed, i.e., the so-called percolation transition (Sansavini et al. 2009).

Although attempts have been made to include simplified models of electric power flow and to capture power flow redistributions, critics concluded (Hines et al. 2010) that evaluating vulnerability in power networks using purely topological metrics can be misleading; (Verma et al. 2015) echoed that context-independent centrality measures underestimate their vulnerability. Attempts have also been made to adopt the CNT-approach to study coupled infrastructure systems and their (physical or logical) interdependencies (Zio and Sansavini 2011).

To better represent the functional behavior of systems driven by physical response to disturbances like the electric power grid, a hybrid approach has been put forward, i.e., agent-based modeling (ABM) of the engineered system coupled to a physical layer. In the ABM approach, each agent is characterized by internal data, its behavior, and its environment and adapts to environmental changes (D'Inverno and Luck 2001). An agent can be used to model both technical components (generators, transmission lines) and non-technical components (human operator); interactions can be organized by messages that are sent to and received by the agents (Schläpfer et al. 2008). The behavioral rules of each agent are represented by finite-state machines and include both deterministic and stochastic time-dependent discrete events (see Fig. 4 for the transmission system operator). A multitude of representative event chains (scenarios) are continuously generated by means of power flow calculations.

Such a hybrid approach has, for example, been applied to the Swiss electric power grid to investigate its applicability to a real-world system that has been mapped by 587 interacting agents and phenomenologically modeled by coupled DC

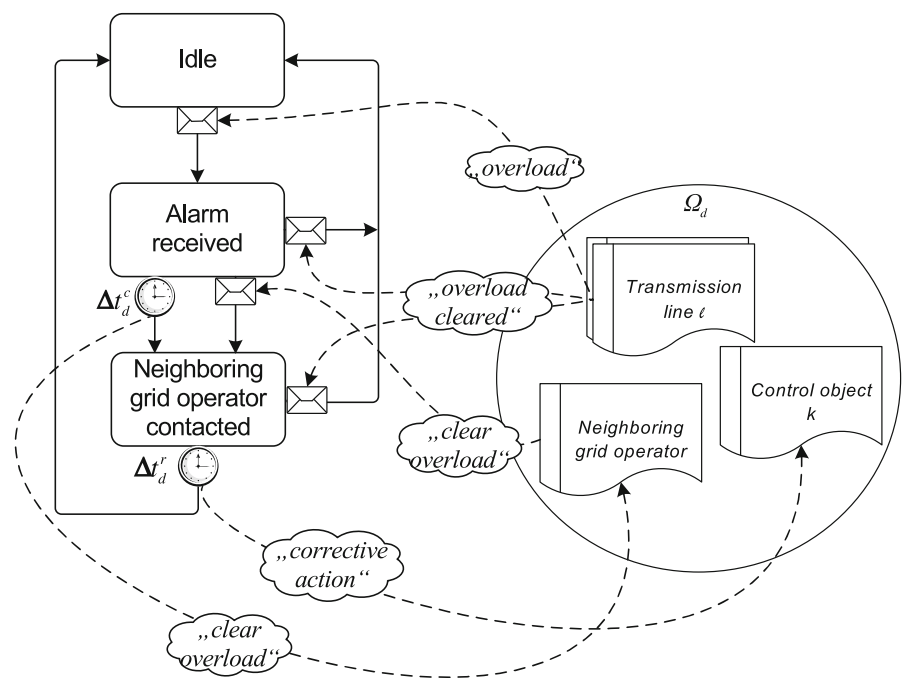

Fig. 4 Finite state machine-here of the transmission system operator (TSO) agent (Kröger and Zio 2011) 

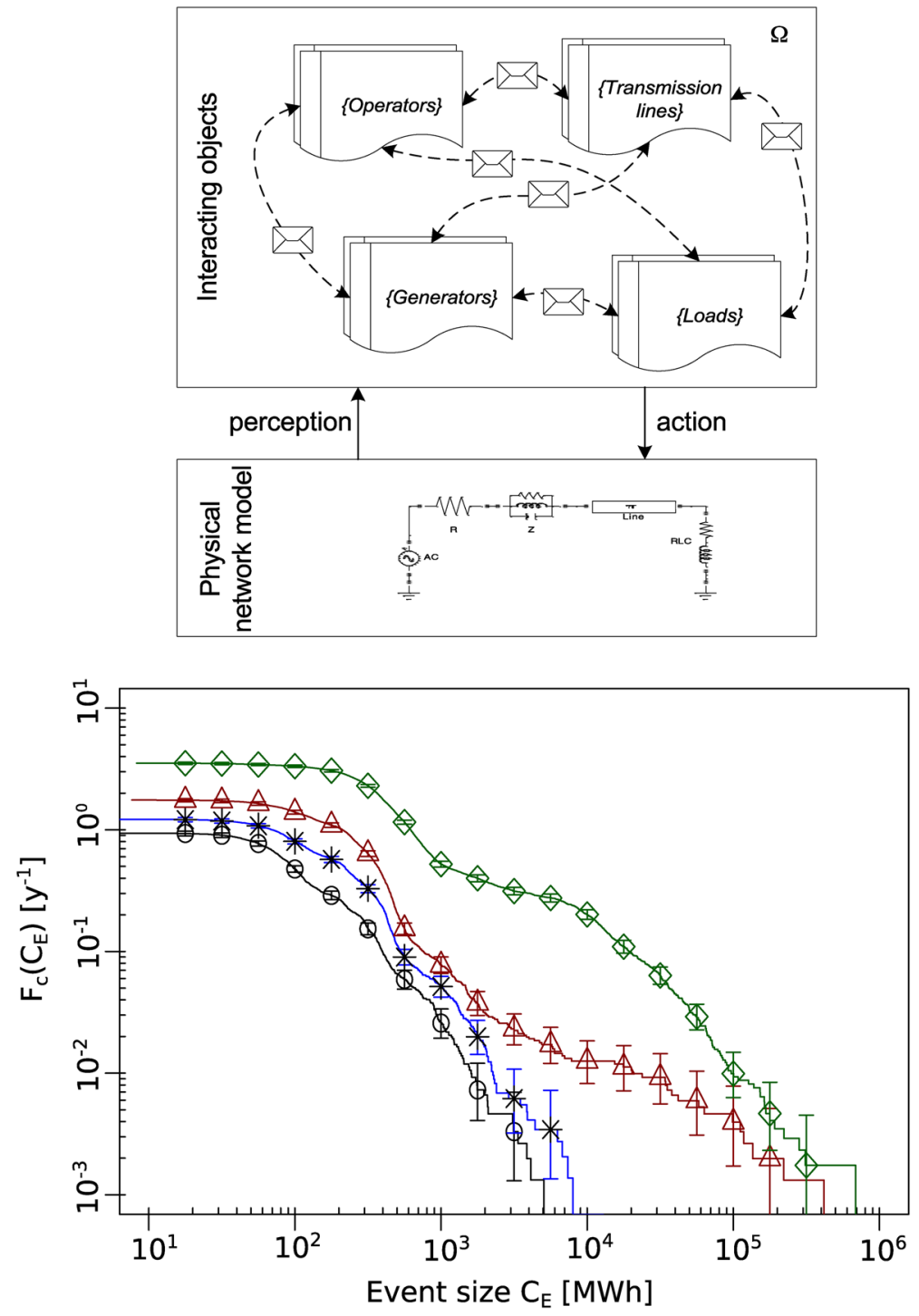

Fig. 5 Hybrid model of Swiss transmission grid (above) and complementary cumulative blackout frequencies (below) for the Swiss power grid and four different system loading levels $L=1.0,1.1,1.2$ and 1.37 (circles, stars, triangles and diamonds) without operator intervention; error bars indicate the $90 \%$ confidence interval (Kröger and Zio 2011)

power flow calculations. The multitude of simulation runs, involving long simulation times, result inter alia in the annual cumulative blackout frequency curve $F_{\mathrm{c}}\left(C_{\mathrm{E}}\right)$, with $C_{\mathrm{E}}$ defined as total unserved energy per event; it follows an exponential distribution as long as the initial load remains within the design level and turns into a power law distribution for higher initial load levels (see Fig. 5). These curves can be significantly influenced by operator response actions, certainly 
within the first (up to) 20 min. Despite major benefits, the hybrid ABM approach suffers from the demand of a large number of model parameters, operational data requirements, and high computational intensity (see also Kröger and Zio 2011); it requires some simplifications and thorough knowledge of the focal system. Maybe these drawbacks explain why this approach has not become popular, yet.

However, these and other modeling approaches, e.g., input-output interoperability modeling (IIM), are still the subject of development within an active field of research. Substantial deficits do exist in the area of human performance assessment which must be integrated into analyses and attempts to secure the operation of socio-technical critical systems like the power grid, as highlighted before. Human reliability analysis (HRA) has evolved in steps, starting in the nuclear field but expanding to other areas; the main objectives of HRA are (Wreathall et al. 2003) (a) to ensure that key human interactions are systematically identified, analyzed, and incorporated into the safety analysis in a traceable manner, (b) to quantify the probabilities of their success and failure, and (c) to provide insights that may improve human performance. After thorough review, the cognitive reliability and error analysis method (CREAM) turned out to be the most suitable and widely used technique in the electricity domain (Kyriakidis 2009; Kröger and Zio 2011; Nan and Sansavini 2016).

\section{Resistance to Malicious Attacks}

Only a few attempts have been made to answer the question of how well an electric power system can withstand malicious attacks. According to a recent literature review (Cuffe 2016 (under review)) mostly CNT has been applied using "centrality measures, of greater and lesser electrical sophistication, to identify the (most) vulnerable components within a grid." Along these lines the Swiss power grid has been modeled by 242 nodes for substations, loads, and generators and by 310 links for lines, with the node size correlated to the degree centrality. Important elements were identified by centrality analysis using deterministic attacks targeted on substations and stochastic attacks on lines (randomly disconnected). A quasi-dynamic load flow model was used to analyze the system's response and cascades in particular (Bilis et al. 2013). The results were not alarming as they showed good safety margins of the Swiss grid: no unstable conditions emerged from the attack on the most critical substation (hub), the effect of cascading failures was very small, and transmission lines were overloaded in only a few scenarios. Recently, planner-attacker-defender models are adopted to develop decisions that co-optimize investment/operating costs and functionality loss after attacks against power systems (Fang and Sansavini 2017). The model bridges long-term system planning for transmission expansion and short-term switching operations in reaction to attacks. More sophisticated studies are needed to develop attack scenarios, apply realistic consequence models, and adequate counteractive measures. 


\section{Paradigm Shift to Resilience}

In essence, we have been facing new challenges that are mainly driven by two factors, the increasing coupling strength and the decreasing heterogeneity, both within and between systems. These changes result in pushing large-scale socio technical systems to a critical state, at which point the prediction of low-probability/ high-consequence events with extreme value distribution is not appropriate (IRGC 2016). The traditional concept of prevention and finally designing systems' resistance capacity by "hardening" against specified exogenous and endogenous failures or actions, respectively, appears obsolete and insufficient. New concepts have been asked for and emerged including the concept of resilience, that has been developed and explored in various fields (Thoma et al. 2016), and has gained traction in the field of technical-engineered systems.

Although there is no commonly accepted definition yet, it means the ability of the system to sustain or restore its basic functionality following a risk source or event (even unknown) (Society for Risk Analysis (SRA) 2015). To elaborate, we define resilience of a system as the ability to resist/absorb the adverse effects of a disruptive force (either sudden (shock) or creeping) with decreasing performance but without collapsing, and the ability and speed it is able to recover and return to an appropriate functionality - by adapting through self-organization and learning and eventually bouncing back or transforming into a different state (see Fig. 6 for illustration). The understanding and assessment of resilience are still in their infancy. In the electric power sector the application of this concept helps to identify best strategies to restore system operation and minimize performance losses after a disruptive force has occurred, often unexpectedly; it also allows for the quantification of trade-offs between investing in system robustness or investing in system recovery.

Attempts are ongoing to further develop the resilience concept and make it operational as well as to define performance metrics and work on recommendations on how to increase the resilience including security of current and future sociotechnical critical systems, e.g., the huge respective program of the Singapore ETH

\section{Paradigm Shift to Resilience}

\section{Four essential patterns}

- Absorbing a shock with decreasing performance, without collapsing

- Recovering from a shock

- Adapting through self-organization and learning

- Bouncing back or transforming into a different state by altering structures or functions and feedback loops

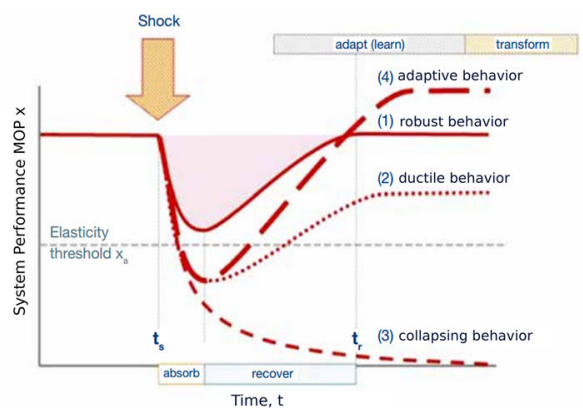

Fig. 6 Illustration of the concept of resilience and patterns of response behaviors (Nan et al. 2014) note: time and performance axis may use different scales 
Box 2 Guiding principles to increase resilience of power supply systems-compiled by the author

Allocate resource buffers, implement physical and functional redundancy/diversity

Decrease connectivity, perform decoupling strategies (islanding)

Strive for robust grid topology, i.e., balance interconnectedness, identify critical nodes and prevent them from spreading failures, optimize structure (degree, connectivity, hybrid) against random failures and targeted attacks

Balance complexity (avoid too little-too high) as well as automation and human control (keep humans in the loop for the unforeseen)

Design for operation within safety margins, system reorganization in response to external changes

Span hazards/threats and triggered scenarios to all imaginable, notably cyber

Strive for predictability by applying new knowledge and advanced tools, use framework to study interdependencies

Center (SEC), the working group of the project on "Energiesysteme der Zukunft" (ESYS) in Germany. Although it might be too early, some guiding principles have been compiled by the author (see Box 2), demonstrating that improving resilience in a sufficiently holistic, cost effective way is a very challenging objective, often in contrast to recent practice and developments/tendencies.

\section{Conclusions}

Western societies depend on a set of large-scale, wide-area systems which are mostly socio-technical by nature. The electricity supply network is of utmost importance; it is the subject of major changes in both structure and technology and is evolving. Historically good performance created complacency. Nonetheless major breakdowns happened worldwide. They provide evidence of complex grid behaviors and the importance of non-technical triggering factors and put the deterministic best-practice $\mathrm{N}-1$ principle as single security criterion in question. The electric power grid-and its cyber-based monitoring and control system in particular-is deemed to be an attractive target for cyber attacks, including manipulation, and was exposed to a major hacker attack just recently. To secure the operation of such a critical infrastructure we have to better understand potential multiple failures, tripping scenarios and cascades, and underlying mechanisms, all highly dynamic, complex, and hard to tackle. As statistical events are (fortunately) rare we have to rely on advanced models and simulations; some techniques are available but need to be further developed and more rigorously applied. Simplified idealized approaches will still be necessary; however, oversimplification may not account for characteristics of real systems like power grids, often integrated into a system of critical socio-technical systems (see also (Gao et al. 2014). There is no commonly agreed "one-fits-all" methodological framework; the analysis of directed malicious attacks is at an early stage. The concept of resilience seems promising to better cope with uncertainties and "unknowns," but there is still intensive work needed to substantiate the concept and make it of practical use; otherwise, it will degenerate into a buzzword. 


\section{References}

Bilis IE, Kröger W, Nan C (2013) Performance of electric power systems under physical malicious attacks. IEEE Syst J 7(4):854-865

Council of the European Union (2008) COUNCIL DIRECTIVE 2008/114/EC of 8 December 2008 on the identification and designation of European critical infrastructures and the assessment of the need to improve their protection. http://eurlex.europa.eu/LexUriServ/LexUriServ.do?uri=OJ:L:2008:345: 0075:0082:EN:PDF. Accessed 20 Dec 2016

Cuadra L et al (2015) A critical review of robustness in power grids using complex networks concepts. Energies 8:9211-9265

D'Inverno M, Luck M (2001) Understanding agent systems. Springer, Berlin

ENTSOE (2006) Final report system disturbance on 4 November 2006. ENTSOE Publication Platform. https://www.entsoe.eu/fileadmin/user_upload/_library/publications/ce/otherreports/Final-Report20070130.pdf. Accessed 20 Dec 2016

European Network and Information Security Agency (ENISA) (2016) Annual activity report. ENISA Publication Platform. https://www.enisa.europa.eu/publications/corporate/enisa-annual-activityreport-2015. Accessed 20 Dec 2016

European Network of Transmission System Operators for Electricity (ENTSOE) (2004) Continental Europe operation handbook. ENTSOE, Brussels

Eusgeld I, Kröger W (2008) Towards a framework for vulnerability analysis of interconnected infrastructures. In: Proc. PSAM9, May 18-23, Hong Kong, pp 107-16

Fang Y, Sansavini G (2017) Optimizing power system investments and resilience against attacks. Reliab Eng Syst Saf 159:161-173

Gao J, Liu Y, D’Souza RM, Barabási AL (2014) Target control of complex networks. Nature Commun 5:5415

Hines P, Cotilla-Sanchez E, Blumsack S (2010) Do topological models provide good information about electricity infrastructure vulnerability? Chaos: an Interdisciplinary. J Nonlinear Sci 20:033122

IRGC (2016) Resource guide on resilience. EPFL International Risk Governance Center, Lausanne, V29$07-2016$

Kröger W, Sansavini G (2016) Principles of disaster risk reduction. In: Platzer K (ed) Handbook of protecting electricity networks from natural hazards. OSCE, Vienna, pp 11-56

Kröger W, Zio E (2011a) Agent-based modeling and simulation. Vulnerable Syst. Springer, Berlin, pp 129-143

Kröger W, Zio E (2011b) Human reliability analysis. Vulnerable systems. Springer, Berlin, pp 157-193

Kröger W, Zio E (2011c) Exemplary application. Vulnerable systems. Springer, Berlin, pp 137-142

Kyriakidis MA (2009) A study regarding human reliability within power system control rooms. Laboratory for Safety Analysis, D-MAVT, ETH Zurich

Nan C, Sansavini G (2016) Developing an agent-based hierarchical modeling approach to assess human performance of infrastructure systems. Int J Ind Ergon 53:340-354

Nan C, Sansavini G (2017) A quantitative method for assessing resilience of interdependent infrastructures. Reliab Eng Syst Saf 157:35-53

Nan C, Sansavini G, Kröger W, Heinimann HR (2014) A quantitative method forassessing the resilience of infrastructure systems. In: Proceedings of the 12 th probabilistic safety assessment \& management conference (PSAM12)

Pagani AG, Aiello M (2013) The power grid as a complex network: a survey. Physica A 392:2688-2700

SANS Industrial Control Systems (SANS ICS), Electricity Information Sharing and Analysis Center (EISAC) (2016) Analysis of the cyber attack on the Ukrainian power grid. SANS Institute Reading Room Site. https://ics.sans.org/media/E-ISAC_SANS_Ukraine_DUC_5.pdf. Accessed 20 Dec 2016

SANS Institute (2016) State of ICS Security Survey. SANS Institute Reading Room Site. https://www. sans.org/reading-room/whitepapers/analyst/2016-state-ics-security-survey-37067. Accessed 20 December 2016

Sansavini G et al (2009) A deterministic representation of cascade spreading in complex networks. Europhys Lett 87:48004

Schläpfer M et al (2008) Reliability analysis of electric power systems using an object-oriented hybrid modeling approach. PSCC, Glasgow

Society for Risk Analysis (SRA) (2015) SRA glossary. SRA publication platform. http://www.sra.org/ sites/default/files/pdf/SRA-glossary-approved22june2015-x.pdf. Accessed 20 Dec 2016 
Thoma K, Scharte B, Hiller D et al (2016) Eur J Secur Res 1:3

Verma T, Ellens W, Kooij R (2015) Context-independent centrality measures underestimate the vulnerability of power grids. Int J Crit Infrastruct 11(1):62-81

Wikipedia (2003) List of major power outages. Wikipedia Online Page. https://en.wikipedia.org/wiki/ List_of_major_power_outages. Accessed 20 Dec 2016

Wreathall J et al (2003) Human reliability analysis insupport of risk assessment for positive train control. Department of Transportation, John A. Volpe National Transportation Systems Center, US

Zio E (2016) Challenges in the vulnerability and risk analysis of critical infrastructures. Reliab Eng Syst Saf 152:137-150

Zio E, Sansavini G (2011) Modeling interdependent network systems for identifying cascade-safe operating margins. IEEE Trans Reliab 60(1):94-101

Zio E, Sasavini G (2008) A systematic procedure for analysing network systems. Int J Crit Infrastruct 4(1-2):172-184 